\title{
Pattern-Wavelength Coarsening from Topological Dynamics in Silicon Nanofoams
}

\author{
M. Castro, ${ }^{1}$ R. Cuerno, ${ }^{2}$ M. M. García-Hernández, ${ }^{3}$ and L. Vázquez ${ }^{3}$ \\ ${ }^{1}$ Grupo Interdisciplinar de Sistemas Complejos (GISC) and DNL, Escuela Téc. Sup. de Ingeniería (ICAI), \\ Universi dad Pontificia Comillas, E-28015 Madrid, Spain \\ ${ }^{2}$ Departamento de Matemáticas and GISC, Universidad Carlos III de Madrid, E-28911 Leganés, Spain \\ ${ }^{3}$ Instituto de Ciencia de Materiales de Madrid (CSIC), E-28049 Madrid, Spain
}

(Received 21 November 2012; revised manuscript received 19 September 2013; published 7 March 2014)

\begin{abstract}
We report the experimental observation of a submicron cellular structure on the surface of silicon targets eroded by an ion plasma. Analysis by atomic force microscopy allows us to assess the time evolution and show that the system can be described quantitatively by the convective Cahn-Hilliard equation, found in the study of domain coarsening for a large class of driven systems. The space-filling trait of the ensuing pattern relates it to evolving foams. Through this connection, we are actually able to derive the coarsening law for the pattern wavelength from the nontrivial topological dynamics of the cellular structure. Thus, the study of the topological properties of patterns in nonvariational spatially extended systems emerges as complementary to morphological approaches to their challenging coarsening properties.
\end{abstract}

Many natural systems lead to tessellations of space into cellular structures with varying degree of order, as in soap froths, polycrystalline materials, biological tissues, fluid convection, galaxy clusters, or graphene-based networks [1-4]. In these patterns, sharp boundaries separate homogeneous regions with sizes and other statistics which can be characterized, including topological relations between domains, down to near $100 \mathrm{~nm}$ [5].

Frequently, these structures evolve in time, with coarsening properties that have focused large interest, ever since von Neumann's and Mullins' classic works on soap froths [6] and metallic grain boundaries [7]. By coarsening, we mean the increase of a characteristic length scale (say, the lateral size of domains, $\ell$ ) as a power law with time, in such a way that universal behavior occurs [8,9]. This behavior is also found in many other systems, like liquid crystals [10] or epitaxial thin film growth [11], granular matter [12], or social dynamics [13], being one of the central open problems in nonlinear physics.

Coarsening systems can be grouped into two large classes: one which can be described in terms of phase separation [8,9] and another one in which morphological instabilities induce pattern formation with a characteristic size [14] that evolves nontrivially. In the latter class, important general criteria [15] have been put forward recently to predict the type of behavior of $\ell$, from remaining fixed, to (interrupted) coarsening, or spatiotemporal chaos. Progress has been achieved for wide classes of models [11,16], the case of two-dimensional (2D) systems $[17,18]$ which are invariant under arbitrary shifts of the "order parameter" $h \rightarrow h+$ const [19] remaining a challenge (particularly, for nonvariational dynamics). For these, the ensuing cell pattern fills space and can be thought of as a foam, so that the time evolution of its topological properties can be meaningful. While for coarsening systems driven by curvature as in phase separation, topological arguments have enabled determining the dynamics of domain areas exactly [20], as experimentally verified [10], a similar approach has not been pursued for the nonvariational systems just mentioned.

In this Letter, we undertake such an approach. Specifically, we produce a cellular surface pattern at submicron scales by ion-plasma erosion of silicon targets. Indeed, reactive plasma techniques stand out as efficient tools for nanostructuring [21]. This system admits an accurate description [22] by a convective Cahn-Hilliard $(\mathrm{CCH})$ equation [23], which is a paradigmatic model for nonvariational coarsening systems, from step instabilities in epitaxy to dewetting of a thin film flowing down an inclined plane, see, e.g., [24] and references therein. We analyze the topological properties of the cellular pattern, and obtain as a consequence the coarsening law for the domain size. Thus, we show that, for nonvariational pattern-forming systems, a topological study of the dynamics provides a complementary route to geometrical descriptions of the coarsening behavior. Technically, we provide an alternative route to derive the coarsening exponent for the $\mathrm{CCH}$ equation, complementary to other approaches, e.g., through phase-diffusion equations. This might prove of interest in the study of challenging related systems, e.g., of the Kuramoto-Sivashinsy (KS) type [14].

Experimentally, we erode $\mathrm{Si}(100)$ targets with a diameter of 3 inches magnetically confined $\mathrm{Ar}^{+}$ion plasma in a commercial Pfeiffer PLS 500 chamber. The ion pressure is $5 \times 10^{-3} \mathrm{mbar}$, and the source is a radiofrequency Hüttinger operating at 25 Watt, with a frequency of 13.56 MHz. After sputtering, the central part of the wafer was analyzed by atomic force microscopy (AFM) in 
tapping mode with a silicon cantilever. In Fig. 1 (left column), we show the evolution of an initially atomically flat target under these conditions, for increasing irradiation times. A cellular structure of submicron-sized domains forms, and evolves with time, that indeed resembles those observed in foams, soap froths, tissues, or nanoparticle networks [1-4]. The domains are not homogeneous in height. Rather, smooth wells (darker regions) are separated by abrupt boundaries (brighter lines). Hence, the system is three-dimensional although the pattern can be characterized in 2D [25], where it is essentially space filling [26]. Morphological processing [27] of the AFM images allows us to determine the precise shape and in-plane organization of the cells or domains. These arrange in a disordered manner, requiring a statistical analysis of their distribution. Indeed, the topological properties of 2D cellular structures (in general) and foams (in particular) are well characterized by a few elegant relations involving various features of the domain distribution, like average area and average number of sides. Some are formulated independently of time, like, Lewis, Aboav, and Lemaitre's laws, while other incorporate dynamics explicitly, like that of von Neumann and Mullins [1-3]. Thus, Lewis' law relates empirically the average area of an $n$-sided domain, $\bar{A}_{n}$, with the deviations of $n$ from its expected value, $\bar{n}$, through

$$
\bar{A}_{n}=\bar{A}_{6}\left[1+\Lambda \frac{\bar{n}}{\mu_{2}}(n-\bar{n})\right] .
$$

Here, $\Lambda$ reflects correlations between cell area and topology (number of sides) [3], and $\mu_{2}$ is the variance of the distribution of the number of sides, $p_{n}$. Meanwhile, Aboav's law describes topological correlations among different cells by relating the average number of sides $m_{n}$ of cells surrounding an $n$-sided domain, with $n$ [28]
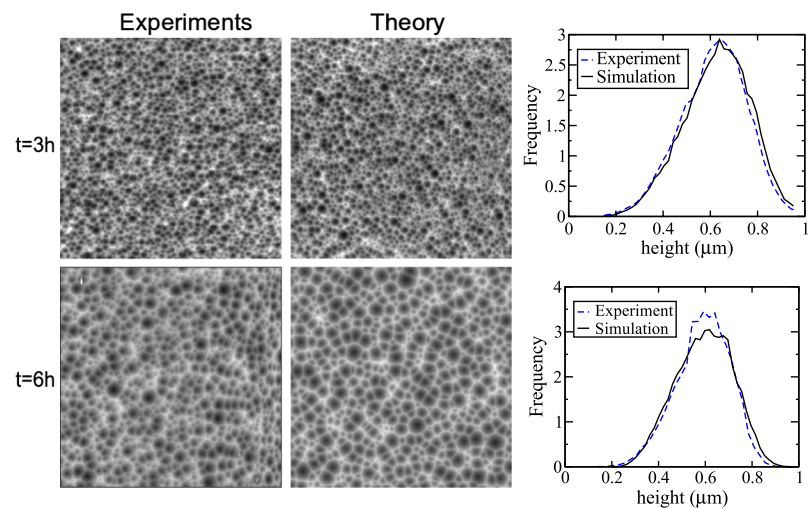

FIG. 1 (color online). Top-view comparison between experimental surfaces (left column) and numerical integration of Eq. (6), central column, at two irradiation times as indicated. The lateral size of all figures is $50 \mu \mathrm{m}$. The right panels show the height distributions for the simulation (black solid line) and experiment (dashed blue line) at the corresponding times.

$$
m_{n}=6-a+\frac{b}{n},
$$

where $a$ and $b$ are numerical constants that depend on correlations in $p_{n}$. In turn, Lemaitre's law relates $\mu_{2}$ with the probability $p_{6}$ for a domain to have exactly six neighbors, different cellular structures, from cracks to biological tissues, corresponding to different points on an universal curve [3]. Although lacking a closed form, it has simple limiting behaviors, see Fig. 2(c):

$$
\begin{gathered}
\mu_{2}=1-p_{6}, \quad \text { if } p_{6}>0.7 \text { (low disorder), } \\
\mu_{2}=\frac{1}{2 \pi p_{6}^{2}}, \quad \text { if } 0.3<p_{6}<0.7 \text { (low order). }
\end{gathered}
$$

Finally, von Neumann-Mullins's (vNM) law incorporates the time evolution of the domain distribution (coarsening) through that of the area of an $n$-sided cell, namely,

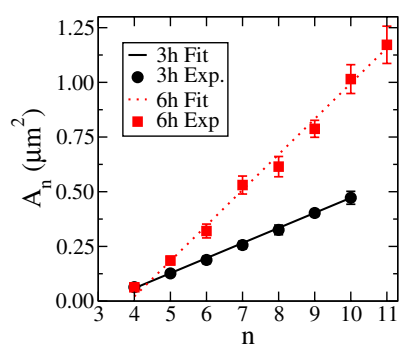

(a)

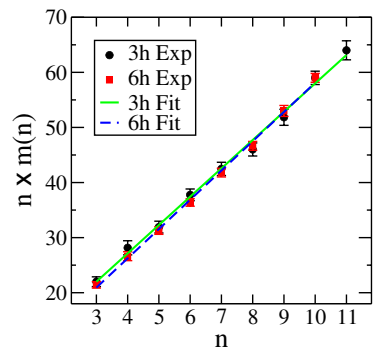

(b)

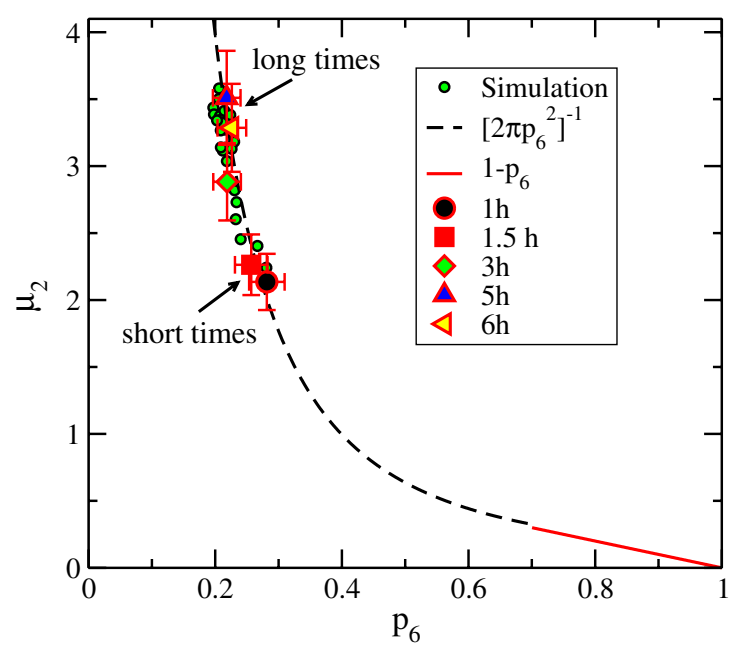

(c)

FIG. 2 (color online). Experimental confirmation of Lewis' (a) and Aboav's (b) laws. For the sake of clarity, we show $n \times m_{n}$ vs $n$. (c) Verification of Lemaitre's law for experiments (large symbols) and simulations of Eq. (6) (small circles). In both cases, points evolve from (more ordered) low $\mu_{2}$-high $p_{6}$ regions to (more disordered) high $\mu_{2}$-low $p_{6}$ regions. At late times in the coarsening regime, simulation points fluctuate, but stay within the same region of the curve. Units are arbitrary if not specified. If not displayed, the error bar is smaller than the symbol size. 


$$
\frac{d A_{n}}{d t}=K(n-6)
$$

where $K$ is a positive constant. This equation has been derived physically for systems in which domain motion is curvature driven, in two [6,7] and three dimensions [29]. Nevertheless, it has been proven widely valid, at least on average, for many other cellular systems [3].

Figure 2 shows the confirmation of Eqs. (1), (2), and Lemaitre's law for our experiments (we discuss the vNM law below). Note that, their range of applicability having been extensively studied [30], frequently these laws are considered statically, except for a few examples [3]. Here, we find that they also hold at different times. In Fig. 2(a), lines are fits of Eq. (1): $\bar{A}_{n}(3 h)=$ $0.067 n-0.205 \quad\{$ regression coefficient $(\mathrm{rc})=0.99951$, reduced $(\mathrm{r})-\chi^{2}=0.15$, see [31], [25] for details $\}$ and $\bar{A}_{n}(6 h)=0.148 n-0.537 \quad\left(\mathrm{rc}=0.99676, \quad \mathrm{r}-\chi^{2}=0.93\right)$. Likewise, in Fig. 2(b), lines are fits to Eq. (2): $m_{n}(3 h)=$ $5.127+6.636 / n\left(\mathrm{rc}=0.998, \mathrm{r}-\chi^{2}=0.62\right)$ and $m_{n}(6 h)=$ $5.282+5.186 / n\left(\mathrm{rc}=0.9991, \mathrm{r}-\chi^{2}=0.68\right)$.

As seen in Fig. 2, Lewis' law fits accurately the data for $4 \leq n \leq 10$. In other systems [5], systematic deviations for all $n$ indicate that physical forces must be considered, besides topological constraints. In our case, the deviations for short and large $n$ are statistical. The validity of Lewis' law is, in principle, restricted to 2D patterns determined solely by the mathematics of space filling. Although the eroded structures are really $3 \mathrm{D}$, the results confirm that the 2D approximation is reasonable. Similarly, Fig. 2(b) shows that the experiments also can be fit by Aboav's law. Line crossing at $n_{c} \simeq 4.2$ in Fig. 2(a) suggests this number, rather than six, as an effective value that separates coarsening from shrinking domains in the vNM law. Similar effects occur, e.g., in soap froths [32]. In our case, they may indicate deviations from curvature-driven cell motion. Also note how, while the mean areas $A_{n}$ change with time (from $3 \mathrm{~h}$ to $6 \mathrm{~h}$ ), the correlations $m_{n}$ do not. Thus, the coarsening process affects the size of the structure, but not so much the correlations among neighboring cells. Regarding the $p_{n}$ distribution, Fig. 2(c) shows that the experiments follow Lemaitre's curve, the structure becoming more disordered with increasing time.

We can formulate a continuum description of the experimental system that incorporates its main dynamical constraints: (i) the net amount of material is not conserved; (ii) the system is isotropic, and (iii) invariant under arbitrary translations, $h \rightarrow h+$ const. Under the morphological instability inducing the experimental cell structure, these constraints lead, via a multiple-scales expansion, to the evolution equation [22]

$$
\begin{aligned}
\partial_{t} h= & -\nu \nabla^{2} h-\mathcal{K} \nabla^{4} h+\lambda(\nabla h)^{2}+\lambda_{2} \nabla^{2}(\nabla h)^{2} \\
& +\lambda_{3}(\nabla h)^{2} \nabla^{2} h,
\end{aligned}
$$

where coefficients $\nu$ and $\mathcal{K}$ are positive and higher order terms are neglected. For $\lambda_{2}=0$, Eq. (6) is an isotropic, twodimensional form of the $\mathrm{CCH}$ equation for which a number of important properties, like the value of the coarsening exponent, have not been obtained previously. Taking simultaneously $\lambda_{3}=0$ yields the KS equation [33].

In the context of ion-erosion models, all terms in Eq. (6) admit physical interpretation [34]. The first term on the right-hand side implements the unstable dependence of the sputtering yield with local curvature. The second term accounts for stabilizing surface-diffusion currents, induced by temperature and irradiation. That with coefficient $\lambda$ reflects the fact that, locally, erosion proceeds along the local normal direction, usually inducing late-time saturation of the amplitude [35]. The $\lambda_{2}$ term arises from local redeposition and surface-confined transport of eroded material [36], while the higher order $\lambda_{3}$ term stems from the interplay between the latter mechanisms and irreversible erosion [36].

All coefficients appearing in Eq. (6) can be determined using an inverse method, as shown in [22]. After rescaling to set the coefficients of the linear terms to unity, we obtain $\lambda=-0.05, \lambda_{2}=0.04, \lambda_{3}=-0.05$, implying that, to nonlinear order, surface-confined transport and irreversible erosion are equally important for the surface dynamics. In Fig. 1 (central column), we show top views of the numerical integration of Eq. (6) (starting with a small amplitude random initial condition), showing good quantitative agreement with the experimental AFM images with respect to the full 3D shape of the morphologies. Further comparison is enabled by the height distributions $\mathcal{P}(h)$ and the radial average of the power spectral density (PSD), $S(q, t)=\langle h(\mathbf{q}, t) h(-\mathbf{q}, t)\rangle$, where $h(\mathbf{q}, t)$ is the Fourier transform of the surface height. We compare the experimental and model behavior of $\mathcal{P}(h)$ (Fig. 1) and $S(q, t)$ (Fig. 3) at several times during the system dynamics.

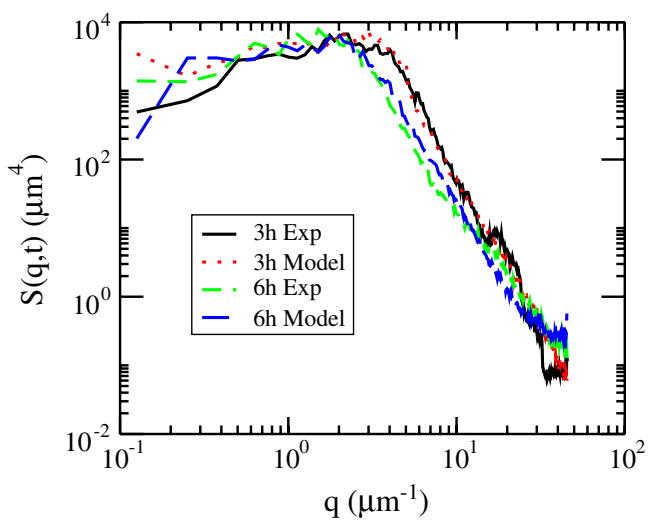

FIG. 3 (color online). Radially averaged PSD, $S(q, t)$, for experiments and model Eq. (6) at two different irradiation times, as shown in the legend. Agreement is obtained, except for $q<3 \mu \mathrm{m}^{-1}$, at which surface positions are uncorrelated. Data for $q>30 \mu \mathrm{m}^{-1}$ may show tip convolution and aliasing effects. 
Agreement indeed allows us to consider Eq. (6) as an evolution equation for the experimental foam.

The shift in the main broad peak of the PSD to small $q$ values for increasing time relates with coarsening of the cell size. Pursuing this morphological description usually requires use of direct approaches [15-18] based on the multiple-scales properties of the interface equation, Eq. (6). Here we turn, rather, to a comparative study of the topological properties of experiments and model, from which the coarsening properties will be derived. Note, in principle we do not know a priori the dynamic law controlling the evolution of the cell structure, which is a collective property of the highly nonlinear Eq. (6). Reports are available on nanostructure formation by reactive plasma techniques [21] in which their space ordering is assessed by topological analysis like Voronoi tessellations [37,38]. However, in our case, cells are not introduced for the analysis, but rather are evolving physical objects. Further, no prior connection seems available between topological properties and morphological ones for pattern-forming systems like the $\mathrm{CCH}$ equation.

We process $[25,27]$ the simulation images of the cell structure using the exact same procedure as employed for the experimental topographies. A compact summary of results is in Fig. 2(c), where the time evolution of the experimental domain distribution encoded by Lemaitre's law is seen to be closely matched by the simulations. During the evolution, cells become wider on average, and increasingly disordered. In Fig. 4(a), we show the time evolution of the mean cell area. For intermediate times $t \geq 3 \mathrm{~h}$, it grows linearly; thus, the typical domain size scales with time as $l \sim t^{1 / 2}$, and the coarsening exponent equals $1 / 2$ [39]. This exponent value can be obtained combining Lewis', Lemaitre's, and von Neumann-Mullins' laws with the substitution $6 \rightarrow n_{c}=4.2$. The space filling condition implies that [40]

$$
\sum_{n} A_{n} p_{n}=\bar{A}=\frac{A_{\text {tot }}}{N}
$$

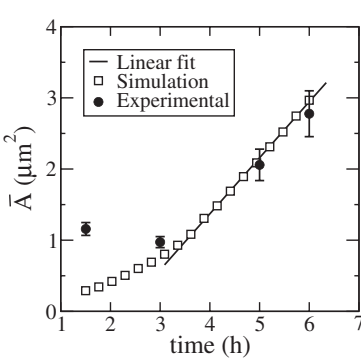

(a)

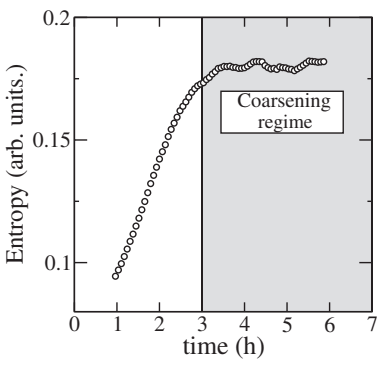

(b)
FIG. 4. (a) Time evolution of the mean cell area, $\bar{A}(t)$. After an initial transient, the system enters the coarsening regime [which, as predicted by Eq. (9), scales linearly with time]. (b) Topological entropy, Eq. (10), vs time. The region in which coarsening takes place is the same as the region where the entropy reaches its maximum stationary value. where $A_{\text {tot }}$ is the total area and $N$ is the number of cells. Taking time derivatives and using Eqs. (5) and (7),

$$
\frac{d \bar{A}}{d t}=\sum_{n} K\left(n-n_{c}\right) p_{n}+\sum_{n} A_{n} \frac{d p_{n}}{d t} .
$$

From Fig. 2(c), we see that, after an initial transient, the distribution of sides evolves into a time-independent form earlier than the area distribution [after $3 \mathrm{~h}$ both the experimental as well as the simulation points fluctuate around the same values of $p_{6}$ and $\mu_{2}$, meaning that $p_{n}(t)$ hardly changes]. Thus, we can assume that $d p_{n} / d t \simeq 0$ and hence, Eq. (8) reduces to the simple coarsening law

$$
\frac{d \bar{A}}{d t}=K\left(\bar{n}-n_{c}\right) \Rightarrow \bar{A} \sim t,
$$

in agreement with Fig. 4(a). For 2D grain growth, the final equality in Eq. (9) is known as the "parabolic law". It was proven by Mullins [7,41] under a scaling assumption on the cell-boundary velocity. However, as noted above, in our experiments and model we do not know a priori what the dynamics of the cells are. Hence, our result can be interpreted as support for an evolution of the cell sides driven by mechanisms, not necessarily curvature, that are consistent with Mullins' scaling.

As in Mullins' proof [41], we are imposing a steady state condition on $p_{n}(t)$. Additional support for this assumption comes through the entropy of the distribution of sides as (alternative definitions can be employed [42]):

$$
S=-\sum_{n} p_{n} \log p_{n} .
$$

Actually, Lewis' law can be derived from a variational principle according to which the entropy of the structure is maximized under area conservation, space filling, and other topological constraints (for instance, that the mean number of sides is six) [40]. At steady state, $S$ reaches a constant maximum value. In Fig. 4(b), we plot the entropy for Eq. (6). Clearly, it is maximized at long times where coarsening takes place, justifying our steady-state assumption. Note that this topological description of coarsening becomes quite different from (albeit complementary to) that based on phase-diffusion equations [11,16-18]. In the latter, the coarsening exponent is determined by the rate of scape of the system from solutions to the corresponding stationary equation. Topologically, it becomes, rather, an expression of the cell distribution having reached a steady state with a maximum entropy.

In summary, we have shown that foamlike structures can be obtained at submicron scales in a system in which the dynamical process differs from soap froths or metallic foams, not being necessarily controlled by curvature-driven domain growth. Physically, morphology does seem to arise as an interplay between irreversible erosion and surface-confined transport. We have obtained a continuum 
description of the cellular structure through an evolution equation that is strongly nonvariational, thus related with a class of coarsening systems that remain a theoretical challenge in the wide context of pattern formation. In turn, the dynamical study of the topological properties of the cellular structure allows us to derive the value of the coarsening exponent. Thus, while a number of assumptions remain to be understood from a more fundamental or rigorous point of view, our Letter puts forward the use of topological characterizations to classify pattern dynamics far from equilibrium, in particular, for classes of systems that remain not completely understood, such as the KS and related systems [43]. An additional benefit of the approach, to be explored further, is its applicability to higher dimensional foams $[29,44,45]$.

We thank R. Gago for discussions. This work has been partially supported by MICINN and MEC (Spain) via Grants No. FIS2009-12964-C05-01, No. FIS2009-12964C05-03, No. FIS2012-38866-C05-01, No. FIS2012-38866C05-01-05, No. MAT2011-27470-C02-02, and No. CSD2009-00013.

[1] J. Stavans, Rep. Prog. Phys. 56, 733 (1993).

[2] D. Weaire and S. Hutzler, The Physics of Foams (Oxford University Press, Oxford, 2001).

[3] G. Schliecker, Adv. Phys. 51, 1319 (2002).

[4] Z. Chen, W. Ren, L. Gao, B. Liu, S. Pei, and H. Cheng, Nat. Mater. 10, 424 (2011).

[5] P. Moriarty, M. D. R. Taylor, and M. Brust, Phys. Rev. Lett. 89, 248303 (2002).

[6] J. von Neumann, Metal Interfaces (American Society for Testing Materials, Cleveland, 1952), p. 108.

[7] W. Mullins, J. Appl. Phys. 27, 900 (1956).

[8] A. J. Bray, Adv. Phys. 43, 357 (1994).

[9] L. F. Cugliandolo, Physica (Amsterdam) 389A, 4360 (2010).

[10] A. Sicilia, J. J. Arenzon, I. Dierking, A. J. Bray, L. F. Cugliandolo, J. Martínez-Perdiguero, I. Alonso, and I. C. Pintre, Phys. Rev. Lett. 101, 197801 (2008).

[11] C. Misbah, O. Pierre-Louis, and Y. Saito, Rev. Mod. Phys. 82, 981 (2010).

[12] B. Andreotti, A. Fourriere, F. Ould-Kaddour, B. Murray, and P. Claudin, Nature (London) 457, 1120 (2009).

[13] C. Castellano, S. Fortunato, and V. Loreto, Rev. Mod. Phys. 81, 591 (2009).

[14] M. Cross and H. Greenside, Pattern Formation and Dynamics in Nonequilibrium Systems (Cambridge University Press, New York, 2009).

[15] P. Politi and C. Misbah, Phys. Rev. Lett. 92, 090601 (2004).

[16] M. Nicoli, C. Misbah, and P. Politi, Phys. Rev. E 87, 063302 (2013).

[17] C. Misbah and P. Politi, Phys. Rev. E 80, 030106 (2009).

[18] S. Biagi, C. Misbah, and P. Politi, Phys. Rev. Lett. 109, 096101 (2012).

[19] Here, we will interpret $h(x, y)$ as the height of an evolving surface above a two-dimensional substrate.
[20] J. J. Arenzon, A. J. Bray, L. F. Cugliandolo, and A. Sicilia, Phys. Rev. Lett. 98, 145701 (2007).

[21] K. Ostrikov, Rev. Mod. Phys. 77, 489 (2005).

[22] M. Castro, J. Muñoz-García, R. Cuerno, M. M. GarcíaHernandez, and L. Vázquez, New J. Phys. 9, 102 (2007).

[23] A. A. Golovin, A. A. Nepomnyashchy, S. H. Davis, and M. A. Zaks, Phys. Rev. Lett. 86, 1550 (2001).

[24] M. A. Zaks, A. Podolny, A. Nepomnyashchy, and A. A. Golovin, SIAM J. Appl. Math. 66, 700 (2006).

[25] See Sections 1.1 and 1.2 in the Supplemental Material at http://link.aps.org/supplemental/10.1103/PhysRevLett .112 .094103 for further details on the image processing algorithm used to quantify the experimental and model morphologies.

[26] Even in the case of soap froths, it is not well established yet whether the system is purely, or effectively, two dimensional; see S. J. Fey, and P. M. Larsen, Curr. Opin. Chem. Biol. 5, 26 (2001).

[27] P. Soille, Morphological Image Analysis: Principles and Applications (Springer-Verlag New York Inc., New York, 2003).

[28] H. Hilhorst, J. Phys. A 39, 7227 (2006).

[29] R. MacPherson and D. Srolovitz, Nature (London) 446, 1053 (2007).

[30] I. Zsoldos, T. Réti, and A. Szasz, Comput. Mater. Sci. 29, 119 (2004).

[31] P. R. Bevington and D. K. Robinson, Data Reduction and Error Analysis for the Physical Sciences (McGraw-Hill, New York, 1969), Vol. 2.

[32] J. Stavans and J. A. Glazier, Phys. Rev. Lett. 62, 1318 (1989).

[33] Although the term with coefficient $\lambda_{2}$ does not seem to modify such properties substantially, it does improve quantitative comparison with experiments.

[34] J. Muñoz-García, L. Vázquez, R. Cuerno, J. A. SánchezGarcía, M. Castro, and R. Gago, in Toward Functional Nanomaterials, edited by Z. Wang (Springer, Dordrecht, 2009).

[35] J. Muñoz-García, R. Cuerno, and M. Castro, Phys. Rev. E 74, R050103 (2006).

[36] M. Castro, R. Cuerno, L. Vázquez, and R. Gago, Phys. Rev. Lett. 94, 016102 (2005).

[37] I. Levchenko, S. Kumar, M. M. A. Yajadda, Z. J. Han, S. Furman, and K. Ostrikov, J. Phys. D 44, 174020 (2011).

[38] F. Ratto, T. W. Johnston, S. Heun, and F. Rosei, Surf. Sci. 602, 249 (2008).

[39] The same happens for the second moment $\mu_{2}$. This dependence of $\mu_{2}$ on time has been reported in the literature [32] and referred to as transient behavior. This transient behavior precedes a saturation regime.

[40] N. Rivier, From Statistical Physics to Statistical Inference and Back, (Kluwer Academic, Dordrecht, 1994), p. 77.

[41] W. Mullins, Scr. Metall. 22, 1441 (1988).

[42] R. Blumenfeld and S. Edwards, Eur. Phys. J. E 19, 23 (2006).

[43] M. Castro, R. Cuerno, M. Nicoli, L. Vázquez, and J. G. Buijnsters, New J. Phys. 14, 103039 (2012).

[44] J. Lambert, R. Mokso, I. Cantat, P. Cloetens, J. A. Glazier, F. Graner, and R. Delannay, Phys. Rev. Lett. 104, 248304 (2010).

[45] E. A. Lazar, J. K. Mason, R. D. MacPherson, and D. J. Srolovitz, Phys. Rev. Lett. 109, 095505 (2012). 